\title{
DIAGNOSTIC DE TROUBLE DU DÉFICIT DE L'ATTENTION AVEC OU SANS HYPERACTIVITÉ (TDAH) : PISTES DE RÉFLEXION AU SUJET DES INÉGALITÉS DE PRÉVALENCE ENTRE ÉTABLISSEMENTS SCOLAIRES
}

\author{
Marie-Christine Brault
}

I.N.S.H.E.A. | « La nouvelle revue - Éducation et société inclusives »

2018/3 N 83-84 | pages 99 à 111

ISSN 2609-5211

Article disponible en ligne à l'adresse :

https://www.cairn.info/revue-la-nouvelle-revue-education-et-societeinclusives-2018-3-page-99.htm

Distribution électronique Cairn.info pour I.N.S.H.E.A..

(C) I.N.S.H.E.A.. Tous droits réservés pour tous pays.

La reproduction ou représentation de cet article, notamment par photocopie, n'est autorisée que dans les limites des conditions générales d'utilisation du site ou, le cas échéant, des conditions générales de la licence souscrite par votre établissement. Toute autre reproduction ou représentation, en tout ou partie, sous quelque forme et de quelque manière que ce soit, est interdite sauf accord préalable et écrit de l'éditeur, en dehors des cas prévus par la législation en vigueur en France. Il est précisé que son stockage dans une base de données est également interdit. 


\title{
Diagnostic de Trouble du déficit de l'attention
} avec ou sans hyperactivité (TDAH): pistes de réflexion au sujet des inégalités de prévalence entre établissements scolaires

\author{
Marie-Christine BRAULT, Ph.D* \\ Professeure, département des sciences humaines et sociales \\ Université du Québec à Chicoutimi, Canada \\ Cotitulaire de la chaire de recherche UQAC-Cégep de Jonquière \\ sur les conditions de Vie, la santé, l'adaptation et les aspirations des jeunes (VISAJ)
}

Résumé: Peu d'études se sont intéressées au diagnostic de Trouble du déficit de l'attention avec ou sans hyperactivité (TDAH) en tant qu'inégalité pouvant être créée par l'école. Pourtant, l'importance du milieu scolaire et des enseignants dans l'identification et l'étiquetage des élèves sous la catégorie TDAH est certaine (Brancaccio, 2000; Hinshaw et Scheffler, 2014), bien que peu étudiée (Conrad et Singh, 2018). Une manière de comprendre et d'éventuellement réduire les faux-diagnostics de TDAH est d'étudier le contexte scolaire, d'où semblent émerger des variations de catégorisation entre les établissements et les enseignants (Fabiano et al., 2013). À partir d'une perspective sociologique, combinée au courant de recherche sur l'effet-établissement, l'objectif du présent article est de proposer des pistes de réflexion concernant la distribution inégale duTDAH et de sa médication entre établissements scolaires.

Mots-clés: Culture scolaire - Effet-établissement - Environnement scolaire - Prévalence - Trouble du déficit de l'attention/hyperactivité (TDAH).

Diagnosis of Attention-Deficit/Hyperactivity Disorder (ADHD) and associated medication intake : Some thoughts on between schools inequalities of prevalence

Summary: Few studies have examined the diagnosis of Attention Deficit/Hyperactivity Disorder (ADHD) under the lens of school inequalities. Yet the importance of the school context and teachers in identifying and labeling students under the ADHD category is certain (Brancaccio 2000, Hinshaw \& Scheffler 2014), although not much is known (Conrad \& Singh 2018). One way to understand and possibly reduce the misdiagnosis of ADHD is to study the school context, where variations in ADHD prevalence are emerging between schools and teachers (Fabiano et al., 2013). From a sociological perspective, combined with school-effect research, the objective of this article is to propose some lines of thought concerning the unequal distribution of ADHD and its medication between schools.

Keywords: Attention Deficit/Hyperactivity Disorder (ADHD) - Prevalence - School culture - School effect School environment.

*mcbrault@uqac.ca 


\section{MISE EN CONTEXTE}

Les inégalités scolaires persistent, malgré les efforts pour les contrer. En raison de leurs caractéristiques sociales et socioéconomiques ainsi que de celles de l'établissement scolaire fréquenté, les élèves ne possèdent pas tous les mêmes opportunités de réussite, de parcours et d'expériences scolaires. Ces inégalités touchent même les processus de catégorisation scolaire (classes spéciales, élèves à risques, etc.). Celles-ci se présentent au plan de l'identification, de l'étiquetage et de la gestion des élèves qui posent problèmes en regard de leur appartenance socioculturelle, mais aussi de leur développement, attitudes et comportements. Le cas du diagnostic de Trouble du déficit de l'attention avec ou sans hyperactivité (TDAH) ne fait pas exception.

Obtenu généralement durant les premières années du primaire, le diagnostic de TDAH est considéré comme le trouble mental le plus prévalent (5 à $7 \%$ ) chez les enfants (Willcutt, 2012), surtout chez les garçons (Arnett, Pennington, Willcutt, DeFries et Olson, 2015) et les jeunes de milieux socioéconomiques défavorisés (Russell, Ford, Williams et Russell, 2016). Ceux-ci sont identifiés suite à une manifestation intense, fréquente et inappropriée des comportements d'inattention et/ou d'hyperactivitéimpulsivité (American Psychiatric Association, 2013). Bien que la vision neurologique et biomédicale du TDAH soit largement et mondialement répandue (Conrad et Bergey, 2014), de plus en plus de voix s'unissent pour rappeler l'importance des aspects sociaux et culturels sous-jacents au diagnostic et à l'identification de ces comportements comme posant problème (Conrad et Singh, 2018; Erlandsson et Punzi, 2017; Graham, 2008; Prosser, 2015; Te Meerman, Batstra, Grietens et Frances, 2017).

L'ampleur des faux-diagnostics de TDAH, estimée à près de $40 \%$ (Chilakamarri et Filkowski, 2011 ; Guay, Lageix et Parent, 2009; Merten, Cwik, Margraf et Schneider, 2017), ainsi que le recours grandissant aux psychostimulants et autres médicaments associés par les enfants d'âge scolaire (Brault et Lacourse, 2012; Vasiliadis et al., 2017) soulèvent plusieurs questions sociales. Largement discuté dans la sphère publique (Frances, 2017 ; Landman, 2015; St-Onge (2013) ; StopMediKids, 2016; Verhoeff, 2010), le surdiagnostic du TDAH constitue un problème social associé à d'importants coûts médicaux et pharmacologiques. Aux États-Unis, par exemple, I'utilisation inappropriée de psychostimulants dans le cas d'un mauvais diagnostic de TDAH est estimée entre 320 et 500 millions US\$ (Elder, 2010). Sur le plan individuel, un faux diagnostic de TDAH peut mener à une consommation non nécessaire de médicaments, ainsi qu'à des effets secondaires (retards de croissance, perte d'appétit, trouble de sommeil), à une stigmatisation associée à une perte d'estime de soi, à des attentes plus faibles de la part des enseignants et à des expériences et des parcours scolaires moins positifs (Thomas, Mitchell et Batstra, 2013). Ces conséquences ont des répercussions à long terme pour les jeunes et affectent autant leur cheminement personnel et professionnel, que relationnel (Thomas et al., 2013).

En reconnaissant certains symptômes associés au TDAH et en fournissant des évaluations comportementales aux médecins, l'école et l'enseignant participent 
activement à l'identification et à l'étiquetage des élèves sous la catégorie TDAH (Brancaccio, 2000; Hinshaw et Scheffler, 2014; Kieling et al., 2014 ; Malacrida, 2004 ; Phillips, 2006; Singh, 2006). Pourtant, très peu d'études se sont attardées à ce diagnostic en tant qu'inégalité pouvant être créée par l'école. Par conséquent, le rôle des écoles et des enseignants dans l'accès au diagnostic et à son traitement pharmacologique est encore peu compris (Conrad et Singh, 2018). Une manière de comprendre et d'éventuellement réduire les faux-diagnostics, de même que le surdiagnostic, de TDAH est d'étudier le contexte scolaire, d'où semblent émerger des variations de catégorisation entre les établissements et les enseignants. À partir d'une perspective sociologique, combinée au courant de recherche sur l'effet-établissement, l'objectif du présent article est de faire une synthèse de la littérature concernant la distribution inégale duTDAH entre établissements scolaires et de proposer des pistes de réflexion au phénomène.

\section{DIAGNOSTIC DE TDAH : DÉVIANCE ET MÉDICALISATION}

Les comportements d'hyperactivité, d'impulsivité et d'inattention sont communs chez les enfants, mais leur intensité et leur fréquence sont variables. À prime abord, la présence de ces comportements chez les enfants n'est pas pathologique: même chez ceux qui démontrent une activité plus forte que la moyenne, il ne s'agit que de différences individuelles. C'est le contexte social et normatif qui transforme ces comportements en maladie et qui les désigne sous un registre de diagnostic pathologique (Conrad et Schneider, 1992).

Dans la perspective sociologique privilégiée ici, les comportements liés auTDAH sont conceptualisés comme des comportements déviants (Conrad et Schneider, 1992). La déviance, définie selon Becker (1963/1985, p. 88), n'est pas une propriété inhérente à certains actes ou certaines personnes, mais plutôt un processus collectif s'effectuant entre un individu et un groupe social. Ainsi, pour qu'un comportement soit jugé déviant, une norme doit avoir été transgressée et cette transgression doit être jugée déviante par un groupe social. Ce jugement peut mener au processus d'étiquetage (Rist, 1997) dans lequel un individu ou un groupe social appose une étiquette à un individu jugé déviant par autrui. Cette déviance peut mener à l'étiquetage des élèves d'abord dans le contexte scolaire, ensuite dans le contexte médical où le diagnostic psychiatrique devient une étiquette légitimée par le pouvoir médical suite à un processus de médicalisation (qui rend médical ce qui ne l'est pas d'emblée) (Conrad et Schneider, 1992). Les processus menant à l'étiquette ne forment pas un consensus, mais dépendent plutôt des contextes sociaux (Rist, 1997): ce que l'on qualifie de déviant change selon les époques et les normes en vigueur, mais également selon l'environnement social. À cet égard, les différences entre environnements scolaires sont importantes et comme le rappelle Mehan (1992, p. 340), ce sont les pratiques institutionnelles que l'on doit observer et non pas les jeunes déviants eux-mêmes. Partant de ce constat, l'étude des différences inter-écoles dans ces pratiques pourrait permettre de mieux comprendre les raisons sous-jacentes à l'identification et l'étiquetage des élèves sous la catégorie TDAH. 


\section{EFFET-ÉTABLISSEMENT}

L'utilisation du courant de recherche sur l'effet-établissement (Dumay, 2004) est intéressante pour étudier les inégalités scolaires. Ce champ considère le caractère unique et distinct de chaque établissement, forgé grâce à une combinaison de caractéristiques spécifiques provenant de deux éléments-clés. D'abord, les éléments structurels, permanents et peu flexibles, représentent les environnements physique et organisationnel de l'école (par exemple, éléments architecturaux, particularités administratives, taille de l'école). L'élément structurel le plus significatif est généralement la composition sociale, définie par les caractéristiques collectives des élèves composant l'école (par exemple, niveau socioéconomique, habiletés académiques, appartenance ethnique, etc.). Ensuite, viennent les processus scolaires, perçus comme les plus malléables, dynamiques et informels de l'établissement (Janosz, George et Parent, 1998), dont font partie la culture, le climat, les pratiques éducatives et managériales de l'organisation. Les éléments structurels et les processus scolaires s'influencent mutuellement pour former un agencement unique qui permet la distinction des établissements scolaires (Dumay, 2004). De cette unicité découle un effet-établissement signifiant que " toutes les écoles ne se valent pas " (Cousin, 1998, p. 10): certaines contribuent à l'accroissement des inégalités entre élèves, alors que d'autres les réduisent et permettent à leurs élèves de réussir au-delà de ce qui était prévu par leurs caractéristiques initiales (Bressoux, 1994). À un niveau plus proximal, on identifie également un effet-enseignant qui relève à la fois des caractéristiques personnelles et sociales des enseignants, que de leurs pratiques pédagogiques (Bressoux, 1994). Un avantage propre à cet angle de recherche est qu'en montrant l'établissement scolaire et l'enseignant comme créateurs potentiels d'inégalités, l'impact des facteurs socioéconomiques et du fardeau de responsabilités incombant aux individus et leur famille est allégé. L'identification de milieux et d'enseignants plus axés que d'autres à l'identification des élèves sous la catégorie TDAH permettra de relever les caractéristiques liées au processus et pourra permettre la réduction des inégalités en ce sens.

\section{Effet-établissement et diagnostic de TDAH}

Par le passé, des différences entre établissements scolaires ont été observées chez les élèves sur le plan académique (Bressoux, 1994 ; Cousin, 1998), comportemental (Milkie et Warner, 2011), émotionnel (Brière, Pascal, Dupéré, et Janosz, 2013) et physique (Miyazaki et Stack, 2015). Quelques études se sont penchées sur les différences inter-établissements dans l'identification des enfants à besoins particuliers (McCoy, Banks et Shevlin, 2012), toutefois, rares sont celles qui ont spécifiquement porté sur le cas précis du diagnostic de TDAH et de la consommation de médicaments associés. Une recherche de la littérature sur ce thème a permis de recenser une douzaine d'études provenant du Canada (D. Cohen, Clapperton, Gref et Tremblay, 1999; Sgro, 2000), des États-Unis (Fabiano et al., 2013; Glass et Wegar, 2000 ; Havey, Olson, McCormick et Cates, 2005 ; Schneider et Eisenberg, 2006; Stone, Brown et Hinshaw, 2010), d'Angleterre et d'Irlande (Ford, Goodman et Meltzer, 2004; McCoy et al., 2012), d'Afrique du Sud (Kern, Amod, Seabi et Vorster, 2015) et du Qatar (Bradshaw et Kamal, 2014). 


\section{DOSSIER}

Si certaines de ces études ont évalué la prévalence à l'aide de données rapportées par les parents (Schneider et Eisenberg, 2006; Sgro, 2000 ; Stone et al., 2010), la majorité a sondé les enseignants (Bradshaw et Kamal, 2014; D. Cohen et al., 1999; Fabiano et al., 2013; Glass et Wegar, 2000; Havey et al., 2005; Kern et al., 2015; McCoy et al., 2012). Parmi celles-ci, plusieurs (D. Cohen et al., 1999; Fabiano et al., 2013; Glass et Wegar, 2000; Havey et al., 2005) ont fait une distinction entre a) prévalence réelle et b) prévalence souhaitée en demandant aux enseignants de rapporter a) le nombre d'élèves dans leur classe qui, à leur connaissance, ont déjà reçu un diagnostic de TDAH ou un médicament pour le traiter et b) le nombre d'élèves supplémentaires qui bénéficieraient du diagnostic et/ou d'un traitement pharmacologique. Cette question sur la prévalence souhaitée donne un aperçu du nombre d'élèves qui, selon l'enseignant, posent problème en classe. II en ressort que les enseignants ajoutent toujours de nouveaux cas potentiels à ceux diagnostiqués et par conséquent, la prévalence souhaitée par les enseignants est plus élevée que la prévalence réelle identifiée par les études épidémiologiques. Par ailleurs, les établissements où la prévalence réelle est la plus élevée sont également ceux où la prévalence souhaitée est la plus élevée.

Bien que la méthodologie employée soit variée, la majorité de ces études conclut que la prévalence du diagnostic de TDAH et de la consommation de psychostimulants varie d'un enseignant, d'une école et d'une commission scolaire à l'autre. Ces études sont importantes, car en mettant au jour des inégalités de prévalence entre les milieux scolaires, elles viennent questionner le rôle du système et de l'environnement scolaires dans le surdiagnostic de TDAH. Malgré le consensus de I'existence des effets du contexte éducatif (CS, école et enseignant) pour l'identification, la compréhension, l'étiquetage et le traitement des élèves manifestant des comportements d'hyperactivité-impulsivité et d'inattention, les explications proposées par ces études sont rares, peu étoffées et ne tiennent pas compte de l'ensemble des éléments composant l'environnement scolaire. Elles portent principalement sur les éléments structurels de l'environnement scolaire, tels que la taille des classes et la composition sociale de l'école, qui sont rigides et plus difficilement modifiables. Par ailleurs, les constats que l'on pourrait tirer à partir de ces résultats sont faibles, car ils manquent d'appuis ou sont contradictoires en ce qui concerne la composition sociale (Fabiano et al., 2013; McCoy et al., 2012), la langue d'enseignement (D. Cohen et al., 1999), la religion des écoles (Schneider et Eisenberg, 2006; Sgro, 2000), le type d'école (privée/publique) (Bradshaw et Kamal, 2014; Kern et al., 2015), la localisation (Fabiano et al., 2013), la taille des classes (Fabiano et al., 2013; Glass et Wegar, 2000; Havey et al., 2005) et les caractéristiques des enseignants (McCoy et al., 2012; Schneider et Eisenberg, 2006). En guise d'exemple, il est encore impossible de tirer des conclusions sur le lien entre la taille des classes et l'identification des élèves sous la catégorie TDAH. Les classes de plus grandes tailles sont à la fois associées positivement (plus d'identification (Havey et al., 2005)) et négativement (moins d'identification et de consommation de médication (Fabiano et al., 2013)) au TDAH. Des résultats contradictoires sont également rapportés pour la composition socioéconomique de l'école: si certains constatent une absence de relation (Ford et al., 2004; Stone et al., 2010), d'autres 
observent que les enseignants dans les écoles défavorisées sont plus (McCoy et al., 2012) ou moins (Fabiano et al., 2013) susceptibles d'identifier des élèves avec un TDAH. Cette absence de consensus appelle à de nouvelles études qui permettront de mieux comprendre le rôle des variables structurelles dans l'identification des élèves sous la catégorie TDAH. Ces études pourraient, par exemple, mesurer autrement la composition sociale de l'école. Conceptualisée principalement sur le plan socioéconomique dans les études antérieures (Fabiano et al., 2013; Ford et al., 2004; McCoy et al., 2012), elle pourrait s'avérer plus fortement liée à la présence d'un TDAH si elle était mesurée par la composition sociale de genre (\% de garçons; \% de filles) et académique (résultats antérieurs des élèves), facteurs réputés être plus fortement liés à la présence d'un diagnostic de TDAH (Schneider et Eisenberg, 2006).

\section{Effet-établissement et diagnostic de TDAH : Au-delà des explications structurelles}

Mettre davantage l'accent sur les processus scolaires (pratiques, culture, climat, etc.) pour comprendre les inégalités de prévalence du TDAH entre établissements scolaires pourrait également être profitable aux recherches futures, car l'étiquetage des élèves est, en soi, un processus (Rist, 1997). Une étude ayant exploré cette voie tend à confirmer I'hypothèse: les écoles ayant une forte proportion d'élèves avec un TDAH se distinguaient significativement des autres, non pas au niveau de la performance scolaire ou du statut socioéconomique, comme on aurait pu le penser, mais plutôt par leurs pratiques éducatives et manageuriales (Stone et al., 2010). Ces écoles accueillaient une moindre proportion d'élèves de minorités ethniques, mettaient davantage l'accent sur les aspects négatifs (mauvais comportements, mauvais résultats scolaires), favorisaient des pratiques pédagogiques préconisant les tests et recouraient davantage à la rétention (redoublement) des élèves (Stone et al., 2010). Les processus scolaires de ces écoles semblent mettre l'accent sur les déficits des élèves, ce qui pourrait venir renforcer l'utilisation de catégories diagnostiques, telles le TDAH.

Des études supplémentaires sont bien sûr nécessaires pour mieux comprendre le rôle des processus scolaires dans l'émergence des diagnostics de TDAH. À cet égard, nous suggérons qu'elles gagneraient également à accorder une attention particulière aux processus scolaires engendrés par les enseignants eux-mêmes, puisque ces adultes sont directement impliqués dans le processus d'étiquetage des élèves (Brancaccio, 2000). Nous proposons deux processus particuliers, soit la culture d'éducabilité et les cultures de diagnostics.

\section{Culture d'éducabilité}

L'effet Pygmalion et la prophétie autoréalisatrice qui en découle est un de ces processus qui impliquent des enseignants (Jussim, 1986; Rosenthal et Jacobson, 1968) et qui pourrait être associé à la prévalence du TDAH. De manière simplifiée, ce processus stipule que les enseignants développent des attentes à l'égard des élèves et que ces attentes orientent la manière dont les enseignants se comportent avec les élèves. Ces derniers réagissent au traitement de l'enseignant et agissent tel qu'attendu. Les attentes des enseignants portent généralement sur l'éducabilité 


\section{DOSSIER}

des élèves (peuvent-ils apprendre et être enseignés?), de ce qu'ils sont capables d'accomplir et de leur potentiel de bon élève. Elles sont façonnées principalement par les caractéristiques individuelles des élèves, notamment celles qui relèvent de l'idéaltype du bon élève que l'on peut caractériser par 3 dimensions principales (Kornblau, 1982) : 1) des comportements (ex. concentration, aime le travail scolaire) : 2) des caractéristiques cognitives et motivationnelles (ex: intelligent, curieux, etc.) et; 3) des caractéristiques personnelles et sociales adaptées à l'école (ex. calme, confiant, etc.). Les élèves qui présentent les symptômes associés auTDAH et qui ont de la difficulté à s'asseoir, à écouter, à attendre leur tour, à se concentrer (etc.) vont donc à l'encontre de ces idéaux en plus de déranger autant l'enseignement, que l'enseignant et les pairs dans la classe (Prosser, 2008). On voit donc émerger une boucle, où les enseignants formulent des attentes moins favorables à l'égard des élèves manifestant des comportements liés au TDAH; en retour, les élèves étant soumis à des pratiques éducatives moins stimulantes et recevant moins de rétroactions manifestent davantage de comportements scolaires inadaptés (incluant les comportements associés auTDAH), qui à leur tour viennent influencer les attentes des enseignants.

Les attentes sont formulées à l'égard d'un seul élève ou d'un groupe d'élèves. Contrairement aux attentes vis-à-vis d'un seul élève, celles visant un groupe ont un impact plus important, car elles servent de référence pour les attentes au niveau individuel et indiquent les normes à suivre pour agir en élève idéal (Kornblau, 1982). Les attentes envers un groupe sont également communiquées plus directement par les enseignants, car qu'elles soient positives ou négatives, faibles ou élevées, elles sont transmises par les comportements de l'enseignant en classe et par ses pratiques éducatives. Par ailleurs, les attentes envers le groupe peuvent être partagées par les enseignants d'une même école, qui s'influencent les uns les autres et partagent des attitudes, des croyances, des attentes, ainsi que des façons de faire et de penser. Lorsque les attentes à l'égard du potentiel scolaire et éducatif des élèves sont partagées à l'échelle d'un établissement scolaire, elles forment une culture d'éducabilité, c'est-à-dire un type de culture scolaire, qui contribue à I'unicité de chaque école et par conséquent à l'effet-établissement (Van Houtte, 2004). La culture scolaire fonctionne comme un système de contrôle, en prescrivant, et proscrivant, des manières de faire et d'être, en plus d'orienter la compréhension et l'interprétation qui sont faites des problèmes, des situations et des phénomènes. En outre, la culture contribue à forger les jugements que les enseignants se font à propos de leurs élèves (Van Houtte, 2004, 2005).

La culture d'éducabilité est influencée par la composition sociale de l'école, c'est-à-dire par les caractéristiques collectives des élèves de l'école (statut socioéconomique, ethnie, genre, etc.) et par les autres processus scolaires (Agirdag, Van Houtte et Van Avermaet, 2013; Brault, Janosz et Archambault, 2014). À titre d'exemple, les écoles ayant une forte proportion d'élèves issus de milieux défavorisés ou issus de l'immigration sont plus à risque de présenter une culture d'éducabilité faible, voire négative (Agirdag et al., 2013). Ces populations sont également plus à risque de présenter des comportements associés au TDAH (Russell et al., 2016). Notre hypothèse stipule que les comportements associés au TDAH pourraient découler 
d'une prophétie autoréalisatrice, suite au fait d'avoir fréquenté un établissment scolaire où la culture d'éducabilité reflète des attentes faibles à l'égard du potentiel des apprenants et promouvoir des pratiques pédagogiques moins stimulantes. Une culture d'éducabilité moins favorable viendrait jouer sur les catégories mentales que les enseignants utilisent lors des interactions avec les élèves et pourraient par conséquent favoriser les problèmes d'indiscipline des enfants. Ces effets pourraient être plus importants encore chez les plus jeunes élèves, qui sont davantages influençables ou tout simplement plus sensibles aux attentes des enseignants (Rosenthal et Jacobson, 1968).

\section{Cultures de diagnostics}

Parallèlement, des travaux s'intéressent à l'incursion de la sphère médicale dans le champ scolaire et s'attardent aux processus de médicalisation, consistant à rendre médical ce qui ne l'est pas d'emblée. La médicalisation de l'éducation (discutée en profondeur par Cohen (1983), Petrina (2006) et Singh (2006)), a contribué à faire de l'école un véhicule de la santé publique en ouvrant ses portes aux infirmières, psychologues et autres spécialistes de la santé. Un des rôles, non-officiel, des enseignants qui est toujours en vigueur est de contribuer au dépistage des problèmes des élèves (S. Cohen, 1983; Singh, 2006), voire au quadrillage psychiatrique de la population, et à suggérer un diagnostic et un traitement médical qui sera ensuite entériné par un médecin (Castel, 1981; Foucault, 1999). Cette fonction semble même renforcée dans les sociétés contemporaines par la présence de cultures diagnostiques, qui "réfèrent aux nombreuses manières dont les diagnostics psychiatriques sont utilisés par les individus (patients, professionnels, tout le monde) pour interpréter, réguler et médier plusieurs formes de connaissance de soi et d'activités "(Brinkmann, 2016, p. 1). On parle ici des cultures au pluriel, car différentes cultures diagnostiques coexistent, et ce au sein d'un même système scolaire. Une étude sur le TDAH en témoigne: une prévalence élevée de diagnostic a été observée dans l'établissement rapportant une attitude totalement positive et une absence de critique à l'égard du diagnostic de TDAH et adoptant un langage et des stratégies psychologiques et psychiatriques pour gérer les problèmes scolaires. Au contraire, des taux moindres de TDAH, voire I'absence de ce diagnostic, ont été observés respectivement dans des écoles a) où I'attitude positive à l'égard du diagnostic de TDAH est tout de même suivie d'une critique de la culture d'étiquetage et de diagnostic qui prévaut actuellement dans les sociétés contemporaines et b) où le discours neuropsychiatrique est complètement absent, les professionnels valorisant une éducation universelle, car tous les élèves vivent des défis et chacun a besoin de soutien à un moment ou à un autre (Odenbring, Johansson et Hunehäll Berndtsson, 2017).

Ces cultures d'éducabilité et de diagnostics nous semblent importantes à étudier, afin de mieux comprendre le phénomène des inégalités de prévalence du TDAH entre établissements scolaires. II nous semble encore plus essentiel de prendre en compte leur interaction et les effets multiplicateurs qui en découle. D'une part, les élèves qui sont perçus comme moins éducables peuvent faire l'objet d'attentes moins positives de la part de leurs enseignants, qu'ils incorporent et agissent tel qu'attendu, en manifestant d'avantage de comportements liés au TDAH. D'autre 


\section{DOSSIER}

part, parce qu'ils sont qualifiés de moins éducables, les élèves ont des chances accrues d'être médicalisés pour les aider à rencontrer les normes scolaires attendues et prescrites (Morel, 2014). Ils sont identifiés sous des catégories à connotation médicale, et conséquemment orientés vers des solutions médicales et pharmacologiques. Dans ce contexte, la médicalisation contribue à rendre les élèves "plus éducables " et devient un outil de discipline scolaire qui est plus ou moins utilisé selon les milieux scolaires (Ramey, 2015). Étudier la culture véhiculée à l'école, que ce soit la culture d'éducabilité ou de diagnostics, offre une manière de comprendre les schèmes cognitifs que les enseignants utilisent lors des interactions avec les élèves, notamment lors de l'identification et de la désignation d'un élève sous la catégorie TDAH.

\section{CONCLUSION}

L'objectif visé par le présent article était de faire une synthèse de la littérature concernant la distribution inégale entre établissements scolaires du diagnostic de TDAH et des médicaments associés et de proposer des pistes de réflexion au phénomène. Les aspects scolaires desquels relèvent l'identification des élèves sous la catégorie diagnostique TDAH sont encore méconnus. À cet égard, cet article constitue le point de départ d'un projet de recherche plus large visant à mieux comprendre le rôle de l'école et des enseignants dans l'identification des comportements associés auTDAH et l'étiquetage des enfants sous la catégorie TDAH. Un des buts du projet consiste à expliquer les inégalités de prévalence que l'on observe entre les écoles à partir des caractéristiques des environnements scolaires, principalement des cultures scolaires. L'étude des caractéristiques de l'environnement scolaire en lien avec l'attribution du diagnostic de TDAH semble être une voie innovante qui permettra de mieux comprendre l'étiologie sociale du trouble et éventuellement d'améliorer la qualité des diagnostics émis. Cela contribue également à rendre justice à la complexité des êtres humaines et à leur unicité, qui est plutôt masquée lorsque l'on met l'accent uniquement sur les facteurs neurobiologiques duTDAH (Erlandsson et Punzi, 2017). Au final, l'objectif est de contribuer à des pratiques diagnostiques plus justes, à une valorisation du métier d'enseignant et à une expérience scolaire plus équitable chez les élèves.

\section{Références}

Agirdag, O., Van Houtte, M., \& Van Avermaet, P. (2013). School segregation and self-fulfilling prophecies as determinants of academic achievement in Flanders. In S. De Groof \& M. Elchardus (Eds.), Early school leaving and youth unemployment (pp. 46-74): Amsterdam University Press.

American Psychiatric Association. (2013). Diagnostic and statistical manual of mental disorder, 5th Edition.

Arnett, A. B., Pennington, B. F., Willcutt, E. G., DeFries, J. C., \& Olson, R. K. (2015). Sex differences in ADHD symptom severity. Journal of Child Psychology and Psychiatry and Allied Disciplines, 56(6), 632-639. doi:10.1111/jcpp.12337

Becker, H. S. (1963/1985). Outsiders. Étude de sociologie de la déviance. Paris: Métailié. 
Bradshaw, L. G., \& Kamal, M. (2014). Prevalence of ADHD in Qatari School-Age Children. Journal of Attention Disorders, Jan., 1-8. doi:10.1177/1087054713517545

Brancaccio, M. T. (2000). Educational hyperactivity: the historical emergence of a concept. Intercultural Education, 11(2), 165-177.

Brault, M. C., Janosz, M., \& Archambault, I. (2014). Effects of School Composition and School Climate on Teacher Expectations of Students: A Multilevel Analysis. Teaching and Teacher Education, 44, 148-159. doi:10.1016/j.tate.2014.08.008

Brault, M. C., \& Lacourse, É. (2012). Prevalence of prescribed Attention-Deficit Hyperactivity Disorder medications and diagnosis among Canadian preschoolers and school-age children: 1994-2007. Canadian Journal of Psychiatry, 57(2), 93-101.

Bressoux, P. (1994). Note de synthèse. Les recherches sur les effets-écoles et les effets-maitres. Revue française de pédagogie, 108(1), 91-137. doi:10.3406/ rfp. 1994.1260

Brière, F. N., Pascal, S., Dupéré, V., \& Janosz, M. (2013). School environment and adolescent depressive symptoms: A multilevel longitudinal study. Pediatrics, 131(3), e702-e708. doi:10.1542/peds.2012-2172

Brinkmann, S. (2016). Diagnostic cultures. A cultural approach to the pathologization of modern life. London: Routledge.

Castel, R. (1981). La gestion des risques: de l'anti-psychiatrie à l'après-psychanalyse. Paris: Éditions de Minuit.

Chilakamarri, J. K., \& Filkowski, M. M. (2011). Misdiagnosis of bipolar disorder in children and adolescents A comparison with ADHD and major depressive disorder Annals of Clinical Psychiatry, 23(1), 25-29.

Cohen, D., Clapperton, I., Gref, P., et Tremblay, Y. (1999). Déficit d'attentionhyperactivité perceptions des acteurs et utilisation de psychostimulants. Laval: Régie régionale de la santé et des services sociaux.

Cohen, S. (1983). The mental hygiene movement, the development of personality and the school: The medicalization of American education. History of Education Quarterly, 23(2), 123-149.

Conrad, P., \& Bergey, M. R. (2014). The impending globalization of ADHD: notes on the expansion and growth of a medicalized disorder. Social Science and Medicine, 122, 31-43. doi:10.1016/j.socscimed.2014.10.019

Conrad, P., \& Schneider, J. W. (1992). Deviance and medicalization: from badness to sickness (Expanded ed.). Philadelphia: Temple University Press.

Conrad, P., \& Singh, I. (2018). Reflections on ADHD in a global context. In M. R. Bergey, A. M. Filipe, P. Conrad, \& I. Singh (Eds.), Global perspective on ADHD. Social dimensions of diagnosis and treatment in sixteen countries (pp. 376-390). Baltimore: Johns Hopkins University Press.

Cousin, O. (1998). L'efficacité des collèges. Sociologie de l'effet établissement. Paris: PUF.

Dumay, X. (2004) Effet établissement: Effet de composition et/ou effet des pratiques managériales et pédagogiques? Un état du débat. In: Vol. 34. Les cahiers de recherche en éducation et formation. Université de Louvain-la-Neuve, Belgique: GIRSEF. 


\section{DOSSIER}

Elder, T. E. (2010). The importance of relative standards in ADHD diagnoses: evidence based on exact birth dates. Journal of Health Economics, 29(5), 641-656. doi:10.1016/j.jhealeco.2010.06.003

Erlandsson, S. I., \& Punzi, E. (2017). A biased ADHD discourse ignores human uniqueness. Int J Qual Stud Health Well-being, 12(sup1), 1319584. doi:10.108 0/17482631.2017.1319584

Fabiano, G. A., Pelham, W. E., Majumdar, A., Evans, S. W., Manos, M. J., Caserta, D., ...Carter, R. L. (2013). Elementary and Middle School Teacher Perceptions of Attention-Deficit/Hyperactivity Disorder Prevalence. Child \& Youth Care Forum, 42(2), 87-99. doi:10.1007/s10566-013-9194-1

Ford, T., Goodman, R., \& Meltzer, H. (2004). The relative importance of child, family, school and neighbourhood correlates of childhood psychiatric disorder. Social Psychiatry and Psychiatric Epidemiology, 39(6), 487-496. doi:10.1007/s00127004-0782-0

Foucault, M. (1999). Les anormaux: cours au Collège de France (1974-1975). Paris: Gallimard: Seuil.

Frances, A. (2017). Conclusive Proof ADHD Is Overdiagnosed. huffington post.

Glass, C. S., \&Wegar, K. (2000). Teacher perceptions of the incidence and management of attention deficit hyperactivity disorder. Education, 121(2), 412-421.

Graham, L. J. (2008). Drugs, labels and (p)ill-fitting boxes: ADHD and children who are hard to teach. Discourse: Studies in the Cultural Politics of Education, 29(1), 85-106. doi:10.1080/01596300701801377

Guay, M.-C., Lageix, P., et Parent, V. (2009). Proposition d'une démarche évaluative duTDAH. In N. Chevalier, M.-C. Guay, A. Achim, P. Lageix, et H. Poissant (Eds.), Trouble déficitaire de l'attention avec hyperactivité. Soigner, éduquer, surtout valoriser. (pp. 3-16). Québec: PUQ.

Havey, J. M., Olson, J. M., McCormick, C., \& Cates, G. L. (2005). Teachers' perceptions of the incidence and management of attention-deficit hyperactivity disorder. Applied Neuropsychology, 12(2), 120-127. doi:10.1207/s15324826an1202_7

Hinshaw, S. P., \& Scheffler, R. M. (2014). The ADHD explosion: Myths, medication, money, and today's push for performance: Oxford University Press.

Janosz, M., George, P., et Parent, S. (1998). L'environnement socioéducatif à l'école secondaire: Un modèle théorique pour guider l'évaluation du milieu. Revue canadienne de psycho-éducation, 27(2), 285-306.

Jussim, L. (1986). Self-fulfilling prophecies: A theoretical and integrative review. Psychological Review, 93(4), 429-445. doi:10.1037/0033-295X.93.4.429

Kern, A., Amod, Z., Seabi, J., \& Vorster, A. (2015). South African foundation phase teachers' perceptions of ADHD at private and public schools. International Journal of Environmental Research and Public Health, 12(3), 3042-3059. doi:10.3390/ ijerph120303042

Kieling, R. R., Kieling, C., Aguiar, A. P., Costa, A. C., Dorneles, B. V., \& Rohde, L. A. (2014). Searching for the best approach to assess teachers' perception of inattention and hyperactivity problems at school. European Child and Adolescent Psychiatry, 23(6), 451-459. doi :10.1007/s00787-013-0466-y 
Kornblau, B. (1982). The teachable pupil survey: A technique for assessing teachers' perceptions of pupil attributes. Psychology in the Schools, 19, 170-174. doi :10.1002/1520-6807(198204)19:2<170::AID-PITS2310190206>3.0.CO ;2-P

Landman, P. (2015). Tous hyperactif. Paris : Albin Michel.

Malacrida, C. (2004). Medicalization, ambivalence and social control: Mothers' descriptions of educators and ADD/ADHD. Health, 8(1), 61-80.

McCoy, S., Banks, J., \& Shevlin, M. (2012). School matters: how context influences the identification of different types of special educational needs. Irish Educational Studies, 31(2), 119-138. doi:10.1080/03323315.2012.669568

Mehan, H. (1992). Comprendre les inégalités scolaires: la contribution des approches interprétatives.

Merten, E. C., Cwik, J. C., Margraf, J., \& Schneider, S. (2017). Overdiagnosis of mental disorders in children and adolescents (in developed countries). Child and Adolescent Psychiatry and Mental Health, 11, 5. doi:10.1186/s13034-016-0140-5

Milkie, M. A., \& Warner, C. H. (2011). Classroom learning environments and the mental health of first grade children. Journal of Health and Social Behavior, 52(1), 4-22. doi:10.1177/0022146510394952

Miyazaki, Y., \& Stack, M. (2015). Examining individual and school characteristics associated with child obesity using a multilevel growth model. Social Science and Medicine, 128, 57-66. doi:10.1016/j.socscimed.2014.12.032

Morel, S. (2014). La médicalisation de l'échec scolaire. Paris : La Dispute.

Odenbring, Y., Johansson, T., \& Hunehäll Berndtsson, K. (2017). The many faces of attention deficit hyperactivity disorder: Unruly behaviour in secondary school and diagnostic solutions. Power and Education, 9(1), 51-64. doi:10.1177/1757743817693026

Petrina, S. (2006). The medicalization of education A historiographic synthesis. History of Education Quarterly, 46(4), 503-531.

Phillips, C. B. (2006). Medicine goes to school: teachers as sickness brokers for ADHD. PLoS Medicine, 3(4), 433-435.

Prosser, B. J. (2008). Beyond ADHD : a consideration of attention deficit hyperactivity disorder and pedagogy in Australian schools. International Journal of Inclusive Education, 12(1), 81-97. doi:10.1080/13603110701683147

Prosser, B. J. (2015). Attention deficit hyperactivity disorder in Australia: Perspectives from the sociology of deviance. Journal of sociology, 51(3), 596-612.

Ramey, D. M. (2015). The Social Structure of Criminalized and Medicalized School Discipline. Sociology of Education, 88(3), 181-201. doi:10.1177/0038040715587114

Rist, R. C. (1997). Comprendre les processus scolaires: La contribution de la théorie de l'étiquetage. In J.-C. Forquin (dir.), Les sociologues de l'éducation américains et britanniques. Présentation et choix de textes (pp. 299-317). Bruxelles: DeBoeck.

Rosenthal, R., \& Jacobson, L. (1968). Pygmalion in the classroom. The Urban Review, 3(1), 16-20. doi:10.1007/BF02322211

Russell, A. E., Ford, T., Williams, R., \& Russell, G. (2016). The Association Between Socioeconomic Disadvantage and Attention Deficit/Hyperactivity Disorder (ADHD): A Systematic Review. Child Psychiatry and Human Development, 47(3), 440-458. doi:10.1007/s10578-015-0578-3 


\section{DOSSIER}

Schneider, H., \& Eisenberg, D. (2006). Who receives a diagnosis of attention-deficit/ hyperactivity disorder in the United States elementary school population? Pediatrics, 117(4), e601-609. doi:10.1542/peds.2005-1308

Sgro, M. (2000). School board survey of attention deficit hyperactivity disorder: Prevalence of diagnosis and stimulant medication therapy. Paediatric child health.

Singh, I. (2006). A Framework for Understanding Trends in ADHD Diagnoses and Stimulant Drug Treatment: Schools and Schooling as a Case Study. BioSocieties, 1(04), 439-452.

St-Onge, J.-C. (2013). Tous fous. L'influence de l'industrie pharmaceutique sur la société. Montréal (Québec) : Les éditions écosociété.

Stone, S., Brown, T., \& Hinshaw, S. P. (2010). ADHD-Related school compositional effects: an exploration. Teachers college record, 12(5), 1275-1299.

StopMediKids. (2016). Le TDA-H: une dangereuse surmédicalisation? Le Devoir.

Te Meerman, S., Batstra, L., Grietens, H., \& Frances, A. (2017). ADHD: a critical update for educational professionals. Int J Qual Stud Health Well-being, 12(sup1), 1298267. doi: $10.1080 / 17482631.2017 .1298267$

Thomas, R., Mitchell, G. K., \& Batstra, L. (2013). Attention-deficit/hyperactivity disorder: are we helping or harming? BMJ (Clinical Research Ed.), 347, 1-7. doi: $10.1136 / \mathrm{bmj} . f 6172$

Van Houtte, M. (2004). Tracking effects on school achievement: A quantitative explanation in terms of the academic culture of school staff. American Journal of Education, 110(4), 354-388. doi:10.1086/422790

Van Houtte, M. (2005). Climate or culture? A plea for conceptual clarity in school effectiveness research. School Effectiveness and School Improvement, 16(1), 71-89. doi:10.1080/09243450500113977

Vasiliadis, H. M., Diallo, F. B., Rochette, L., Smith, M., Langille, D., Lin, E., ... Lesage, A. (2017). Temporal Trends in the Prevalence and Incidence of Diagnosed ADHD in Children and Young Adults between 1999 and 2012 in Canada: A Data Linkage Study. Canadian Journal of Psychiatry, 62(12), 818-826. doi:10.1177/0706743717714468

Verhoeff, B. (2010). Drawing borders of mental disorders: An interview with David Kupfer. BioSocieties, 5(4), 467-475. doi:10.1057/biosoc.2010.24

Willcutt, E. G. (2012). The prevalence of DSM-IV Attention-Deficit/Hyperactivity Disorder : A meta-analytic review. Neurotherapeutics, 9, 490-499. doi:10.1007/ s13311-012-0135-8) 